\title{
A SURVEY ON DYNAMIC SPECTRUM ACCESS TECHNIQUES IN COGNITIVE RADIO
}

\author{
Pinki Yadav ${ }^{1}$, Subhajit Chatterjee ${ }^{2}$ and Partha Pratim Bhattacharya ${ }^{3}$ \\ ${ }^{1,3}$ Department of Electronics and Communication Engineering \\ Faculty of Engineering and Technology, \\ Mody Institute of Technology \& Science (Deemed University) \\ Lakshmangarh, Dist. Sikar, Rajasthan, Pin-332311, India. \\ ${ }^{2}$ Department of Electronics and Communication Engineering \\ Regent Education and Research Foundation \\ Barackpore, Kolkata, India \\ ${ }^{1}$ pinki.yadav150yahoo.in \\ ${ }^{2}$ chsubhajitchegmail.com \\ 3 hereispartha@gmail.com
}

\begin{abstract}
Wireless networks are characterized by fixed spectrum policy. With increasing demands for wireless communication efficiently using the spectrum resources has become an essential issue. Cognitive radio is a form of wireless communication which is used to sense the spectrum and find the free spectrum. It is used by unlicensed users without causing interference to the licensed user. Cognitive radio with the dynamic spectrum access is key technology which provides the best solution by allowing a group of Secondary users to share the radio spectrum originally allocated to the primary users. Dynamically accessing the unused spectrum is known as dynamic spectrum access (DSA) which becomes a promising approach to increase the efficiency of spectrum usage. In this paper, DSA models are discussed along with different methods such as game theory based method, a measurement-based model, network coded cognitive control channel, Markovian Queuing model, the Delay performance of threshold policies, fuzzy logic based method and spatio-temporal spectrum management model.
\end{abstract}

\section{Keywords}

Wireless communication system, cognitive radio, dynamic spectrum access, fuzzy logic, threshold policies, spectrum management.

\section{INTRODUCTION TO COGINITIVE RADIO}

The ever-increasing demand of the wireless communication applications and services affirms the importance of the effective usage of the limited radio spectrum. Each user is assigned a license to operate in certain frequency bands. Most of the time spectrum remains unused and it is very difficult to find the unused spectrum. The allocated spectrum has not been utilized properly and it varies with time, frequency and geographical locations. For the removal of the spectrum scarcity and the unutilized spectrum band, Cognitive radio and Dynamic spectrum access technology has been introduced.

DOI : 10.5121/ijngn.2012.4403 
Cognitive radio is a figure of wireless communication in which a transceiver can perceive, which communication channels are in use and which are not, and accordingly switch into empty channels while avoiding busy ones. This optimizes the use of available radio-frequency spectrum while minimizing interference to other users. It is a hybrid technology involving software defined radio as applied to spread spectrum communication [1].

The concept of cognitive radio was first officially presented by "Joseph Mitola" III, at the Royal Institute of Technology in 1998 and published later in an article by Mitola and Gerald Q. Maguire, Jr in 1999 [2]:

As defined by Haykins, the cognitive radio is an "intelligent wireless communication system that is aware of its surrounding environment (i.e., outside world) and uses the methodology of understanding by-building to learn from the environment and adapt its internal states to statistical variations in the incoming RF spur by making corresponding changes in certain operating parameters (e.g., transmit-power, carrier-frequency, and modulation strategy) in real-time, with two primary objectives in mind, highly reliable communication whenever and wherever needed; efficient utilization of the radio spectrum" [3]. The FCC (Federal communication commission) ruled in November 2008, in which unused part of RF spectrum (known as white spaces) made available for public use. White Space Device Technology prevents interferences, such as spectrum sensing and geolocations capabilities [1]. The parameter can altered on the basis observations of multiple factors from external and internal environment of cognitive radio environment, like radio frequency spectrum, user behaviour, and network state. Spectrum in 400$1000 \mathrm{MHz}$ range is shown in Figure1 [4].

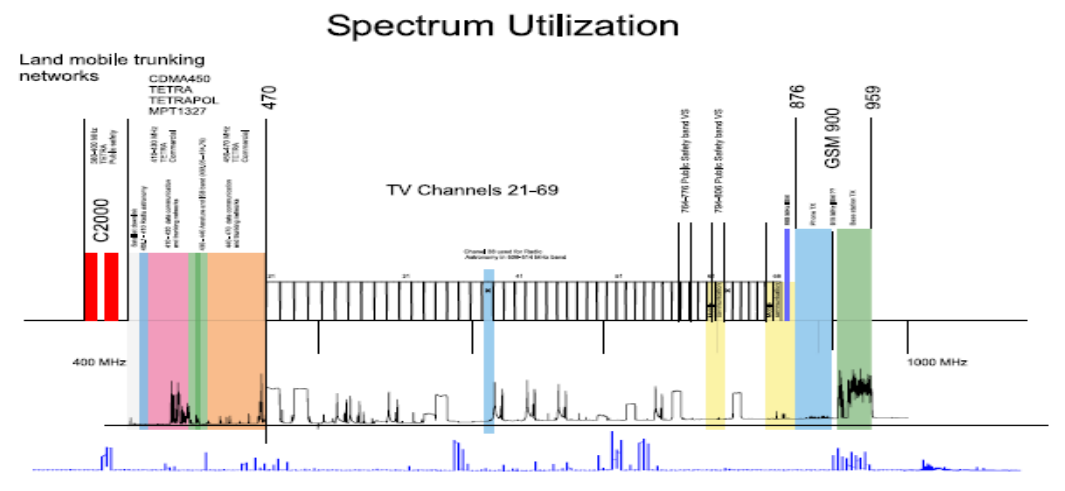

Figure 1 Spectrum utilization

Efficient utilization of spectrum improves by allowing a secondary user (SU) to utilize a licensed band when the primary user (PU) is absent. So the detection of spectrum hole is important as shown in Figure 2 [5]. 


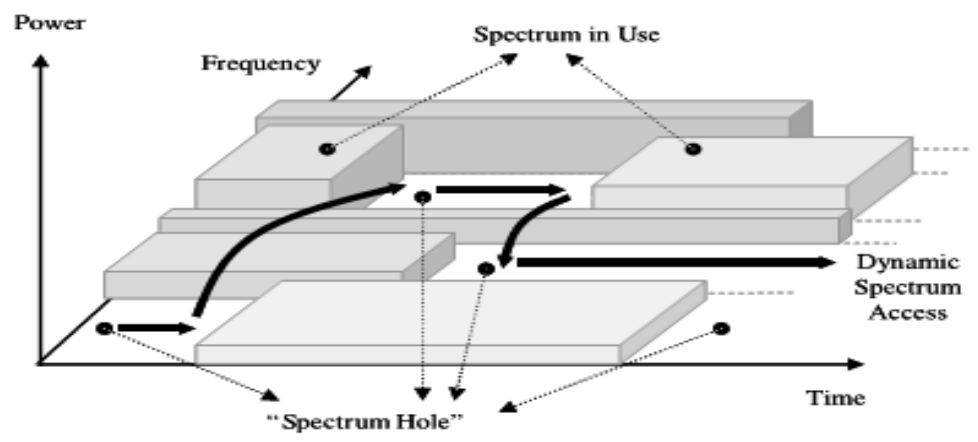

Figure 2 Spectrum hole concept

Spectrum holes is basically a concept which correspond to the latent opportunities for safe use of spectrum i.e. non-interfering and considered as multidimensional regions within frequency, time, and space. The main challenge for secondary radio systems is to be able to robustly sense when they are within such a spectrum hole. To agree to a amalgamated discussion of the core issues in spectrum sensing, the "Weighted Probability of Area Recovered (WPAR)" metric is introduced where the performance of a sensing strategy and the "Fear of Harmful Interference" are evaluated [6]. Cognitive radio is such a inimitable radio technology where user has to decide which part of the spectrum is available. When a user function in a approved (licensed) band (i.e. spectrum sensing), the best available channel is selected (i.e. spectrum management), other users access this channel (i.e. spectrum sharing) and the channel is vacated when a licensed user is detected (i.e. spectrum mobility). Regulatory bodies in various countries (including the Federal Communications Commission (FCC) in the United States, and Ofcom in the United Kingdom) found that most of the radio frequency spectrum was inefficiently utilized. The idea of spectrum sharing is shown in Figure 3 below [7].

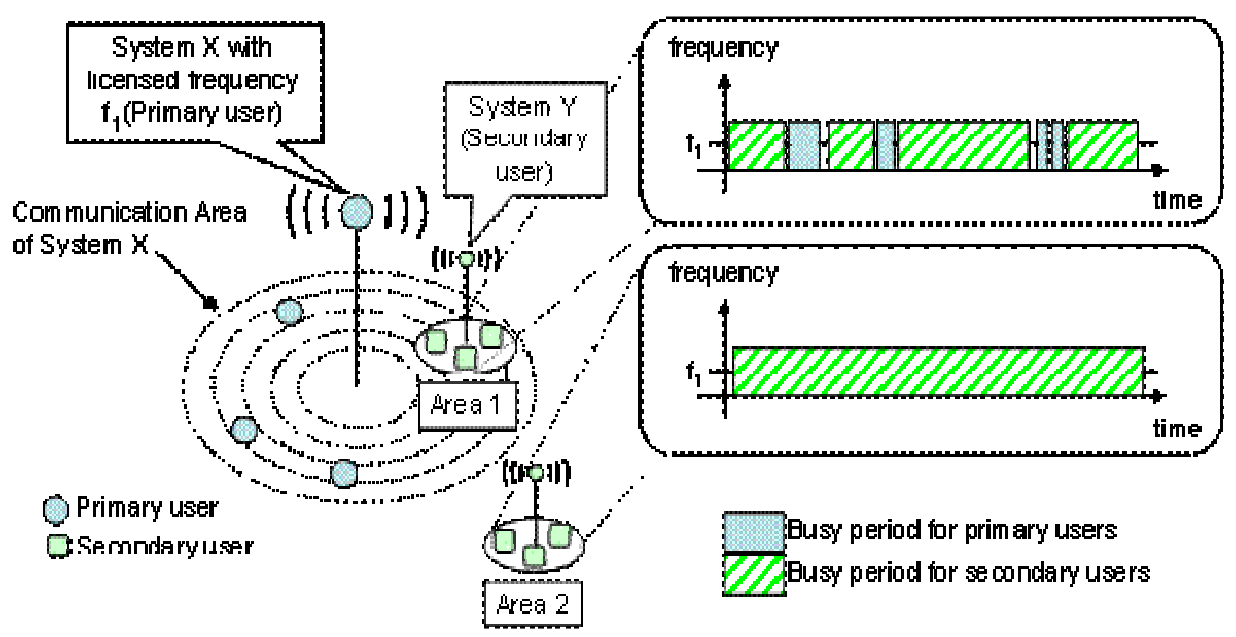

Figure 3 Spectrum Access by Cognitive Radio 
There are two types of cognitive radio, full cognitive radio and spectrum sensing cognitive radio. Full cognitive radio takes into account all the parameters that a wireless node or network can be aware of. Full cognitive radio is also known as "Mitola Radio". Spectrum sensing cognitive radio is used to find channels in the radio frequency spectrum [1].

The radio spectrum animatedly changes the functions in accordance with the surrounding i.e. he radio frequency, transmission power, modulation scheme, communication protocol can be changed by cognitive radio without any alteration of the hardware environment.

The main functions of cognitive radios are: [8]

1) Spectrum Sensing: - Spectrum sensing supports to detect the spectrum holes (Underutilized bands of the spectrum) providing high spectral resolution capability. Spectrum sensing technique classifies into direct and indirect method. Direct method is also known as frequency domain approach in which estimation is carried out directly from signal. Indirect method is known as time domain approach in which estimation is performed using autocorrelation of the signal [9]. Spectrum sensing techniques are classified into three categories:

- Transmitter detection: cognitive radios should be capable of determining a signal (if any) from a primary transmitter which is locally present in a certain spectrum and there are several approaches to perform this and those are namely :

- Matched filter detection

- Energy detection

- Cyclostationary feature detection

- Cooperative detection: Cooperative detection refers to spectrum sensing methods where information from multiple Cognitive radio users is incorporated for primary user detection.

- Interference based detection.

2) Spectrum management: - Spectrum management capturers the best available spectrum to meet user communication requirements. Cognitive Radio decides the best spectrum band to meet the quality of service requirements over all available spectrum bands. Therefore spectrum management functions are important for cognitive radios. Spectrum management functions can be classified into: i) Spectrum analysis. ii) Spectrum decision.

3) Spectrum mobility: - Spectrum mobility is defined as the process when a cognitive radios users exchange its frequency of operation. Cognitive radio network target is to use the spectrum in a dynamic manner by allowing the radio terminals to operate in the best available frequency band and also maintaining seamless communication requirements during the transition to better spectrum.

4) Spectrum sharing: - Spectrum sharing is function of cognitive radio which provides the fair spectrum scheduling method. Spectrum sharing has one of the major challenges open spectrum usage. It can be regarded to be similar to generic media access control (MAC) problems in existing systems. 
Opportunistic use of the radio-frequency (RF) spectrum is enabled by cognitive radio for dynamic spectrum access, where unlicensed users are allowed to utilize licensed bands under the circumstance that interference is as little as possible with the licensed bands. A cognitive radio operates or transmits on bands detected as being free, leaving them whenever a primary user is sensed. [10].

\section{DYNAMIC SPECTRUM ACCESS}

Dynamic spectrum access(DSA) is a technique by which a radio system dynamically adapts to available spectrum holes with limited spectrum use rights, in response changing circumstance and objectives: interference created changes the radio's state, changes in environmental constraints [11]. The main objective of DSA is to overcome two types of interference concern: harmful interference caused by malfunctioning device and harmful interference caused by malicious user. Dynamic spectrum management is also referred to as dynamic spectrum access. DSA that was first demonstrated in 2006 by the Defense Advanced Research Project Agency (DARPA) and Shared Spectrum Company (SSC) of Vienna, VA [12]. In [13], DSA is such an advanced approach to spectrum management which is closely correlated to other management techniques such as flexible spectrum management and spectrum trading. This allows users to access a particular piece of spectrum for a defined time period or defined area which they cannot exceed without re-applying for the resources. A DSA procedure would follow the following steps:-

- Monitor spectrum to see which frequencies have no other radio activity (i.e. they are not being used by anyone).

- Agree with other dynamic spectrum access devices in the network which frequencies will be used, via same previously agreed common channel.

- Being communicating on the agreed frequency band.

- Continue to monitor the spectrum for attempts other user to access this spectrum.

- Change frequency bands and adjust power as necessary.

The monitoring and managing of the radio resources are done by a single device in the network (centrally managed network) or by each of the devices individually and cooperatively (autonomous network).

In this paper, dynamic spectrum access models are discussed in section 2 which helps to improve performance of a communication network as a whole. In section 3, different methods of dynamic spectrum access are discussed.

\section{MODELS OF DYNAMIC SPECTRUM ACCESS:-}

Dynamic spectrum access is a new concept which has overcome the limitations of fixed frequency spectrum assignment and management. Dynamic spectrum access models for cognitive radio can be categorized as dynamic exclusive-use, open sharing model, and hierarchical access model as shown in Figure 4 [14]. 


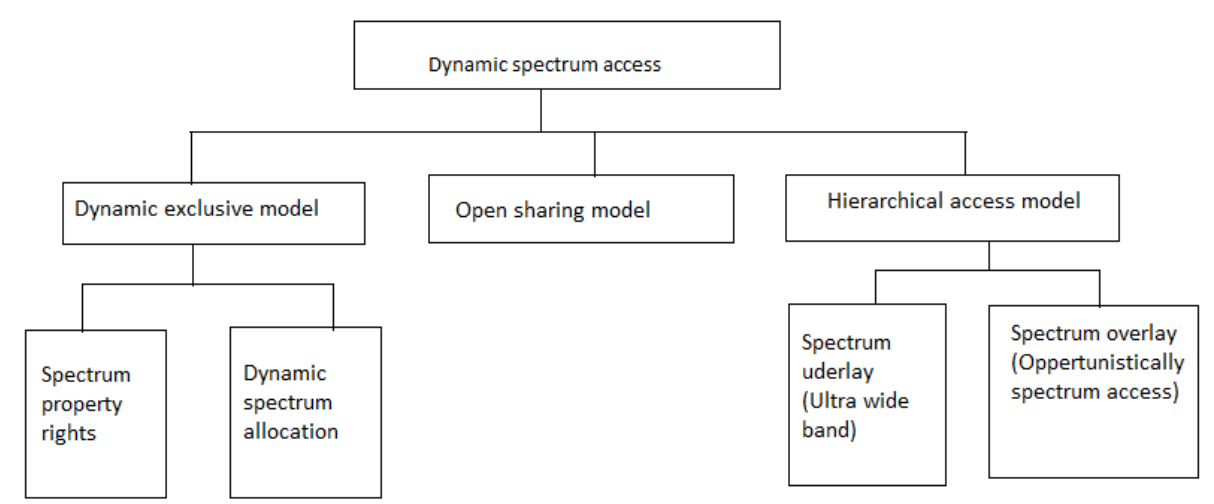

Figure 4 Dynamic Spectrum Access Models

a) Dynamic exclusive use model: - Dynamic exclusive use model maintains the basic structure of the current spectrum regulation policy and Spectrum bands are licensed to services for exclusive use. The main objectives of this model are to improve the flexibility and spectrum efficiency. It manages spectrum in finer scales of time, space and frequency and use dimensions. There are two types of dynamic exclusive use model they are:

i) Spectrum property rights: In this approach licenses are allowed to sell and trade spectrum and to freely choose the technology. The economy and market an important role to play in lashing toward the most gainful use of this limited resource.

ii) Dynamic spectrum allocation: Spectrum efficiency is improved by Dynamic spectrum allocation through dynamic spectrum assignment by using the spatial and chronological traffic statistics of different services. It can be said in other way i.e in a given area and at a given instance, spectrum is allocated to services for elite use. This allocation varies in a much sooner scale than the current policy. Based on an ddedicated-use model, elimination of white space is not possible in spectrum resulting from the burst nature of wireless traffic.

b) Open sharing model: - Open sharing model is also called spectrum commons model. This model uses open sharing among peer-users as the foundation for managing a spectral region. This model is supported from the phenomenal success of wireless services operating in the unlicensed industrial, scientific and medical (ISM) radio band. It has three types [7]: Uncontrolledcommons, Managed-commons, Private-commons.

i) Uncontrolled-commons:-This is also referred as open spectrum access. When a spectrum band is managed no entity has exclusive licensed to the spectrum band. It is maximum transmit power constraint.

ii) Managed-commons:-This represents an effort to avoid the tragedy of commons by imposing a limited form of order or structure of spectrum access.

iii) Private-commons:-Spectrum owner specifies technology and protocol for the CR user access. CR user may receive a command from spectrum owner (transmission parameter). CR user may sense and access the spectrum. 
c) Hierarchical access model: - Hierarchical access model [15], in which radio spectrum can be simultaneously shared between primary (licensed) user and secondary (unlicensed) user. CR users can opportunistically access the radio spectrum if it is not occupied or fully utilized by primary users. There are also two types of model; Spectrum overlay and Spectrum underlay.

i) Spectrum overlay:-The spectrum overlay model actively explored in a going DARAP XG program and advocated by Mitola targets for aggressive opportunistic exploitation of white-space or spectrum "gaps" in spatial-temporal domain [7]. Cognitive Radio will have to identify the idle spectrum band, which are not used by licensed users at a certain time and location and use those idle spectrum bands dynamically to unlicensed users. This model is shown in Figure 5 (a). This model allows primary and secondary transmission. Secondary users can use part of their power for secondary communication and remain part of the power to relay primary transmission, these enabling premises for an overlay system model [16]. Example of spectrum overlay is TDMA, FDMA, and OFDMA system [17].

ii) Spectrum underlay: - Some constraints are imposed by spectrum underlay approach on the transmission power of secondary users so that they are operated well below the noise floor of primary users. By scattering transmitted signals over a wide frequency band (UWB), secondary users can latently achieve a short-range high data rate with very low transmission power shown in Figure 5 (b). In the worst case, it is assumed that the primary users transmit all the time; this approach does not depend on detection and utilization of spectrum white space [14].

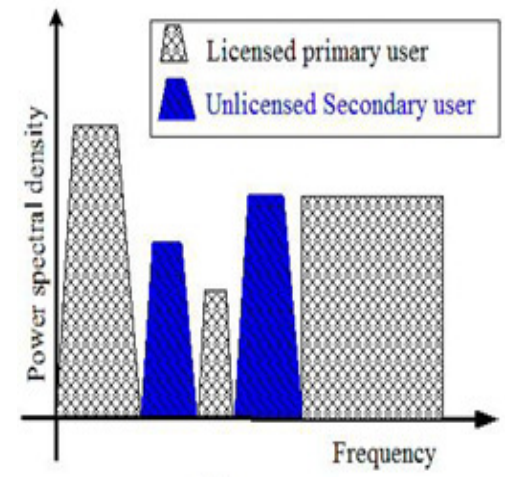

(a)

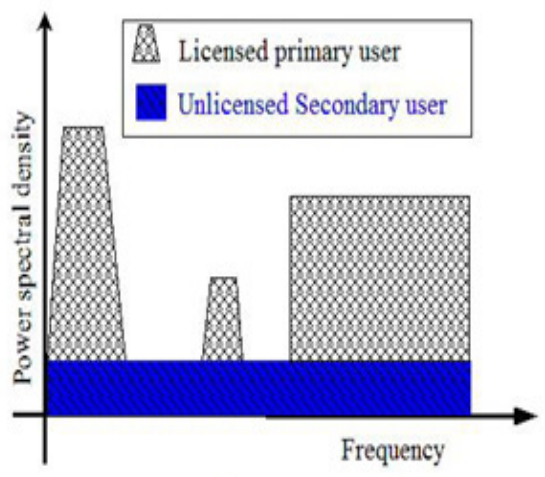

(b)

Figure 5 Spectrum overlay and underlay approaches

In spectrum overlay approach; secondary CR user can transmit with a high transmission power to increase their rates for giving spectrum opportunities however they have to identify the idle frequency bands which are not used by primary users. Similarly, in spectrum underlay approach, the secondary users do not need to identify the spectrum opportunities and can transmit simultaneously coexisting with primary users however they are not allowed to transmit with high transmission power even if the entire RF band is idle (i.e., entire RF is not used by primary users) [17]. Therefore overlay is also known as interference model and underlay is known as interference avoidance model [16].

There are many methods of DSA which work based on these models and discussed below. 
International Journal of Next-Generation Networks (IJNGN) Vol.4, No.4, December 2012

\section{METHODS OF DYNAMIC SPECTRUM ACCESS}

We have discussed different methods of dynamic spectrum access in this section along with their feature.

\section{i) Game theory for cognitive radio networks}

A Game theory is a mathematical tool that analyzed and planned the interaction among multiple decision makers. There are three major components in a strategic-form game model [18]:

1) A finite set of players denoted by $\mathrm{N}$;

2) A set of action, denoted by $A_{i}$, for each player $i$; and

3) Payoff/utility function, denoted by $u_{i}: A \rightarrow R$, which measure the outcome for player $i$ determined by the actions of all player, $A=X_{i} \in_{N} A_{i}$.

In above definition and notations, a strategic game is denoted by $\left\langle\mathrm{N},\left(\mathrm{A}_{\mathrm{i}}\right) ;\left(\mathrm{u}_{\mathrm{i}}\right)\right\rangle$.

Game theoretic spectrum sharing schemes are classified into four parts:

1) Non-cooperative spectrum sharing games: - Nash equilibrium is a solution to understand noncooperative game theory. Nash equilibrium often suffers from extreme competition among selfish players in a non-cooperative game and the outcome of the game is inefficient. In non-cooperative spectrum sharing game with rational network users, each user only cares about his/her own benefit and chooses the optimal strategy that can maximize his/her pay off function and such outcomes of the non-cooperative game is termed as Nash equilibrium.

2) Economic games, auction games and mechanism design: - It can be applied to the economic world to deal with how people interact with each market. The key concept of the game theory is rationality and equilibrium. Often players are the seller and buyer in the market (such as firms, individuals and so on). Payoff functions are defined as the utility or revenue that players want to maximize and equilibrium strategies are considerable interested.

Auction theory is an applied branch of game theory which analyzes the interaction in the auction market and researches. An auction conduct by an auctioneer is a process of buying and selling product by eliciting bid from possible buyers and deciding the auction outcome based on the bids and auction rules. The auction rules or auction mechanism is obtaining from whom the goods are allocated to and how much price they have to pay.

The structure of a game is to design the mechanism and this game structure is "designed" by a game designer called a "principal" who want to choose a mechanism for his/her own interest and resource constraints and incentive constraints are Co equally considered in an allocation problem with private information.

3) Cooperative games: - Cooperative spectrum sharing is a game in which network users have an agreement on how to utilize and distribute the spectrum resources. Two types of cooperative games are i) Bargaining games ii) Coalitional games. 
4) Stochastic games: - A stochastic games is an extension of Markov decision process by considering the interactive competition among different agent. A stochastic spectrum sharing game is such a game in which network users adapt their strategies according the changing environment and other user's strategies. There are many other research challenges such as, Defining a proper payoff function, Efficiency of equilibrium, Issues in mechanism design, Issues in stochastic games, security.

\section{ii) A Measurement-based model for dynamic spectrum access in WLAN Channel}

A Measurement-based model is proposed [19], in which Continuous-time Semi-Markov model is used that captures the WLAN behavior so good enough to be used for deriving optimal control strategies within a decision theoretic frame work. A measurement-based model is based on actual measurements in the 2.4GHZ ISM band using a vector signal analyzer to collect complex base band data. Continuous-time Semi-Markov model is able to capture the data with good accuracy. A measurement setup shown in figure 6. Different from existing publication that use a commercial WLAN adapter card to obtain packet trace but here they use a vector signal analyzer (VSA) to capture the raw complex baseband data. These data have to find busy and idle periods of the channel. In Measurement setup, both WLAN and VSA are provided as shown in Figure 6 respectively.

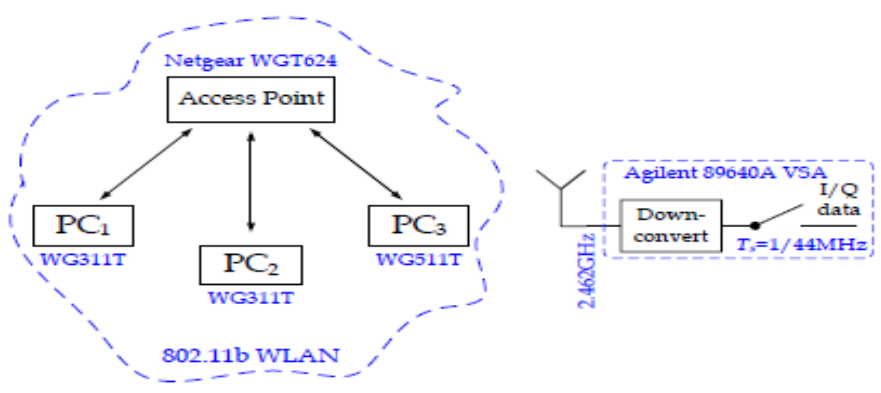

Figure 6 Measurement setup

The WLAN setup consists of a net gear WGT624 wireless router and three computers with wireless adapter card (two net gears WG311T and WG511T). The router operates in a 22MHZ frequency band around $2.462 \mathrm{GHZ}$ (channel 11). The router as well as work stations locates in the same room resulting high signal to noise ratio between nodes and no hidden terminals. Traffic is generated using Distributed Internet Traffic Generator (D-ITG), which allows to statically characterizing parameters such as inter-departure time and packet length. This measurement is based on two traffic scenario. One of the high rate UDP traffic from workstations to the router (the other computers are turned off) and to verify the compatibility of the setup. After capturing the transmission of WLAN, employ Agilent 89640A vector signal analyzer to collect the complex data base band sample. The device internally down converts $2.462 \mathrm{GHZ}$ to an internal IF frequency at a sample rate of $44 \mathrm{MHZ}$. Continuous-time Semi-Markov process in which allows an arbitrary specification of sojourn time distribution in each state. A Semi-Markov process is a stochastic process whose transition behavior characterized in two steps. First step is the transition between states follow a markov chain and specified by transition matrix in Equation 1, where $\mathrm{p}_{\mathrm{ij}}$ denoted the probability that transition from state $\mathrm{i}$ to state $\mathrm{j}$ occur. 
International Journal of Next-Generation Networks (IJNGN) Vol.4, No.4, December 2012

$$
\mathrm{P}=\left[\begin{array}{ccc}
p_{11} & \cdots & p_{1 n} \\
\vdots & \ddots & \vdots \\
p_{n 1} & \cdots & p_{n n}
\end{array}\right]
$$

Secondly, given that the system is in state $i$ and will transition to state $\mathrm{j}$, the sojourn time $\mathrm{t}$ in state $\mathrm{i}$ is distributed according to cumulative distribution function $\mathrm{Q}_{\mathrm{ij}}(\mathrm{t})$. The estimator in Equation 2

$$
\hat{p}_{i j=\frac{n_{i j}}{n_{i}}}
$$

In which the transition count $n_{i j}$ is the number of transitions $i \rightarrow j$ occurring in our observation Sequence. Similarly

$$
n_{i=\Sigma_{i}^{k}} n_{i k}
$$

In Equation 3 is the number of times the system resides in the state. High SNR transmission between nodes and no hidden terminals the sequence of states DATA $\rightarrow$ SIFS $\rightarrow$ ACK is essentially deterministic (the corresponding transition probabilities are very close to one). Hence, it is possible to simplify the model by lumping, these states together. While this model inhibited the occurrence of collisions, retain good accuracy since collisions are infrequent. The Markov model is as shown in Figure 7.
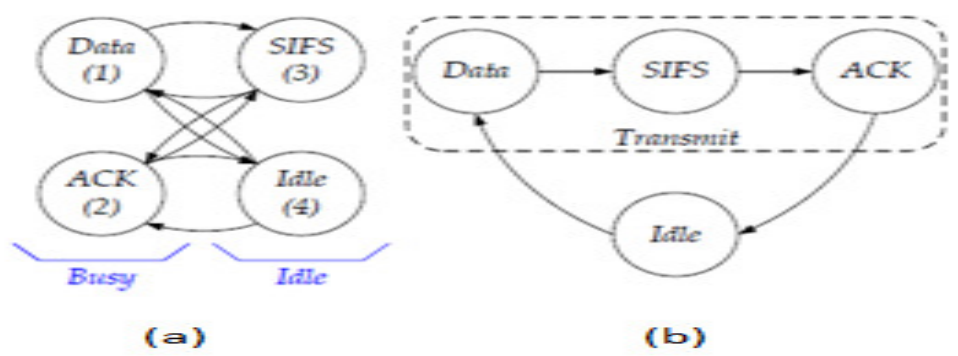

Figure 7 (a) Markov model. (b) The lumped model (with deterministic DATA $\rightarrow$ SIFS $\rightarrow$ ACK transitions is shown on the right)

In Figure 7 'transmit' state (a lumped version of DATA, SIFS, and ACK states with deterministic transitions), and an idle state is shown. The transition probabilities for this simplified semiMarkov model is now trivial, since every transmit state must be followed by an idle period Continuous time Semi-Markov model captures the idle periods remaining between the burst transmissions of the wireless LAN. This model strikes a good compromise between accuracy and computation complexity.

\section{iii) Dynamic Spectrum Access using a network coded cognitive control channel}

Dynamic Spectrum Access using network coded cognitive control channel [20], allows the users to opportunistically and efficiently access the available channel for communication. Important aspects of opportunistic spectrum access are i) Implementation of the control channel. ii) Multi channel medium access control. iii) Primary user detection. iv) Secondary users reuse the unused spectrum of primary uses. In this method all secondary users visit all channels in a pseudo random fashion and exchange control information whenever they happen to meet in any channel. 
The control information exchanged by secondary users consist of all the information which is needed to select switch patterns as well as a resource allocation for data communication, according to a pre-defined deterministic algorithm. This method has important aspects such as, primary user performed over all channels in each allocation period, to track the varying pattern of primary user's activity. The detection information gathered by each during an allocation period is to be disseminated to all users using the control channel. Cooperative detection is carried out by each user using the same deterministic algorithm. The resource allocation algorithm, which is run independently by each user, assigns transmission opportunities only on free channels. This method is completely distributed and it does not need dedicated spectrum resources for control purposes but rather leverages of the virtual control channel which is implementing network coding techniques and exploit a cooperative detection strategy to identify unused spectrum. This method works without the establishment of a control channel, this is achieved by having each secondary user detect the presence of primary activity and decide whether to access the spectrum independently from another user. The main issue is that channel switch pattern needs to cover all channels; so that primary user detection can be performed effectively but the control information cannot be transmitted by secondary users on those channels in which primary users are active. This causes a degradation of the dissemination performance of network coded cognitive control channel-DSA with respect to NC4-MAC. The performance evaluation of NC4-DSA with respect to primary user detection, interference performance, the spectrum reuse efficiency of the secondary users and good put in dynamic spectrum access as shown in Figure 8, Figure 9, Figure 10, and Figure11. In Figure 8, the value of the signal to noise ratio of the primary user is $y \in[0,5] d B$. The normal probability scale is used for both axes; cooperative detection strategy allows to achieve significant improvements in the achievable tradeoff between primary user detection and false alarm probability. The detection improves with increasing $\mathrm{N}_{\mathrm{s}}$ and $\mathrm{S}$.

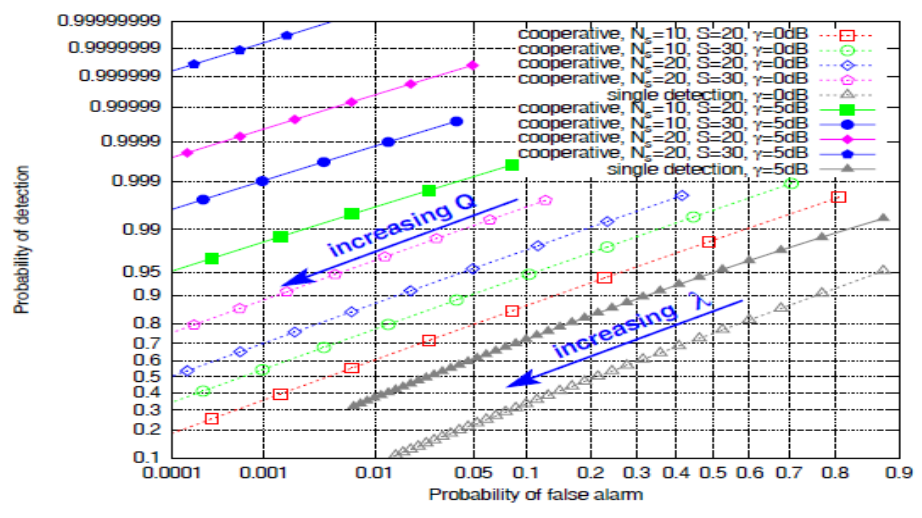

Figure 8 Primary user detection

In Figure 9 and Figure 10, $\mathrm{P}_{\text {inter } \mathrm{f}}$ and $\mathrm{P}_{\text {reuse }}$ is plotted as a function of the mean activity duration $11=1 / \beta$ and inactivity duration $\mathrm{I}_{0}=1 / \alpha$ of the primary user respectively for $\mathrm{c}=10$, $\pi_{1}=0.7$ and $\lambda_{C D}=5 d B$. 


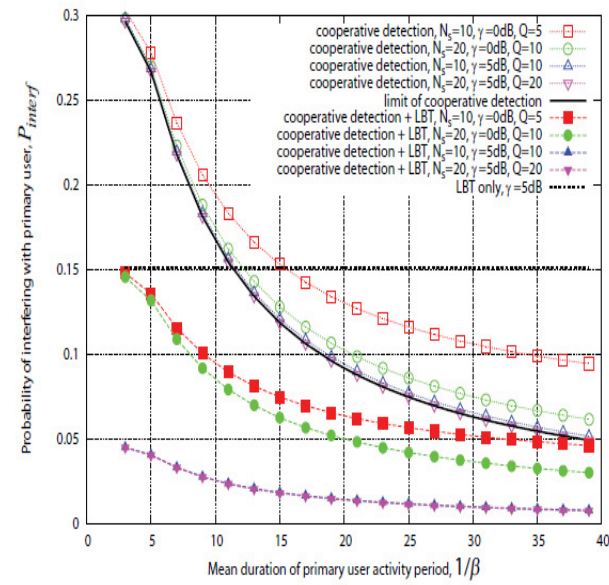

Figure 9 Interference to the primary user caused by secondary users

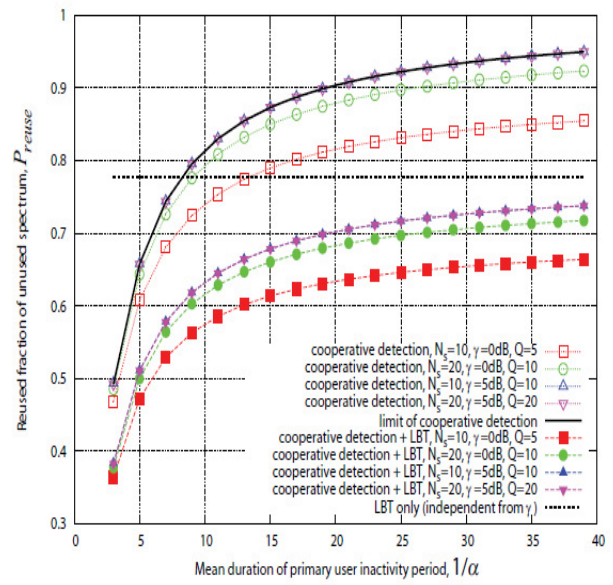

Figure 10Efficiency of secondary user reuse of spectrum unused by primary user

In Figure $9 \mathrm{P}_{\text {inter } \mathrm{f}}$ and $\mathrm{P}_{\text {reuse }}$ are decreases when $\mathbb{l}_{1}$ Increases, since the longer activation period of the primary user allows secondary user to detect it and avoid interfering with it. In the case where $\mathrm{N}_{\mathrm{s}}=10$ and $\gamma=0 \mathrm{~dB}$, probability of a single detection attempt very low due to low SNR of the primary user and benefits of cooperation are limited due to the small number of users. In Figure 10 the probability of reusing unused spectrum depends on the mean duration of the inactive period of the primary user.

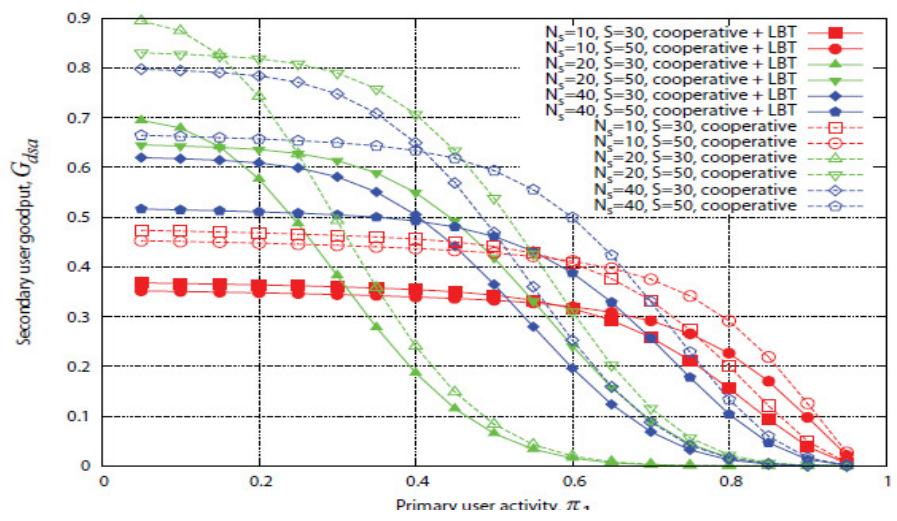

Figure 11Goodput for secondary spectrum access

The Good put the performance of NC4-DSA for both the original "cooperative detection only" version and the "cooperative + LBT" variant. The resulting performance is shown in Figure 11, function of the primary user activity $\pi_{1}$ For $C=10, \gamma=5 \mathrm{~dB}, \lambda_{c D}=5 \mathrm{~dB}, l_{1}=35, T_{a n} / T_{c t r l}=$ 600 , and $\lambda_{L E T}=3 \mathrm{~dB}$ when also LBT is used. Effect on the detection of the primary users and the reuse efficiency of unused spectrum on the overall good put is limited, since detection works almost perfectly with the value of $\mathrm{S}$ that is practical for the dissemination of control information in the primary / secondary scenario; this effect does not increase in $\pi_{1}$. 


\section{iv) Markovian Queuing Model for Dynamic Spectrum Allocation in centralized Architecture}

Markovian Queuing model for dynamic spectrum allocation in which centralized architecture is used. In centralized network the master/controller of this ad-hoc network coordinates spectrum allocation with the surrounding CR in the network. This CR ad-hoc network assumes to coexist with the network of licensed users where the controller of licensed user is updated with the CR coordinating engine. A centralized network eliminates hidden terminal problem and provide better coverage and efficiency spectrum handover technique. Each SU is consisting of two transrecievers, one is dedicated to control and second software defined radio based. The SDR based transrecievers scan the availability of spectra in its vicinity and forward the information of these spectrum holes to the master/controller. In this case, SU form an infrastructure less network and the BS are infrastructure based network. The equivalent model of network queues is shown in Figure12 [21].

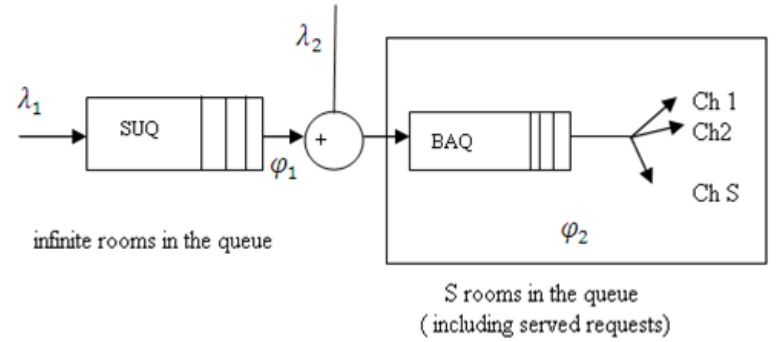

Figure 12 Queuing Model for DSA in Cognitive Radio

These queues are in the special case of stochastic processes, characterized by the arrival process of service requests, waiting list of requests to be processed. The queue stacking all the entries of SUs is referred to secondary user queue and the entire request entering this queue are served on the first come first serve (FCFS) basis. At any time when bandwidth needs to be allocated to the $\mathrm{SU}$, the head considers both the request from the SU and the PU, who need it licensed channel. Therefore while distributing a number of frequencies for PU and SU; the arrival rates of both the users are summed to access the frequencies with the Head. This queue so formed is referred to as bandwidth allocation queue (BAQ). Markov process is used to analyze the Queuing model. The blocking probability $\mathrm{P}_{\mathrm{B}}$ for the bandwidth request made by $\mathrm{CR}$ that finds all the channels with Head as occupied is given by Erlarg-B formula as given below in Equation 4:

$$
P_{B}=\frac{\rho^{s}}{s ! \Sigma_{i=0}^{s} \frac{p^{i}}{i !}}
$$

The Blocking probability is shown in Figure13. Variation in $\mathrm{P}_{\mathrm{B}}$ with respect to change in number of available channels in the system as $2,5,7,10,13$, and 15 . Blocking probability increases with increase in SU traffic in the network. 


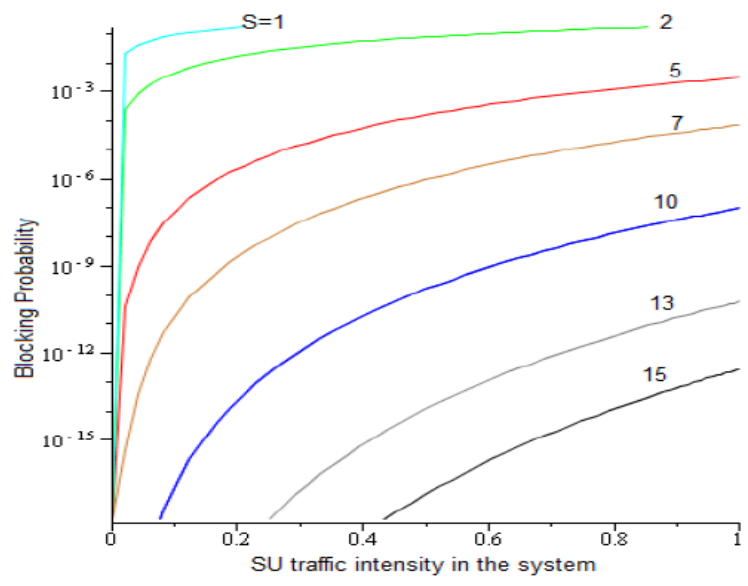

Figure 13 Blocking probabilities $\left(P_{E}\right)$ Against SU utilization in the system $\left(p_{1}\right)$. The Variation in $P_{E}$ is depicted with different numbers of channels (S), available with the system.

\section{v) Delay performance of threshold policies for dynamic spectrum access}

The delay performance of threshold policies for DSA is proposed [22]. A simple time-threshold policy for the SU to minimize the average delay while satisfying the collision probability constraint of the PU (primary user). Such policies perform closely to an optimized policy found by a Markov chain formulation, while facilitating analytical analysis of the delay and collision probability. The time-threshold policies have important aspects which are as follow:

1. The threshold policy performs closely to the optimal policy found by Markov decision process (MDP) formulating, but simpler in structure and provide better insights and being computationally more efficient than the MDP policy.

2. The threshold policies facilitate analytical characterization of the delay and collision probability under general PU is busy and idle distribution.

The time threshold policy with the threshold $\Gamma$ is defined such that the SU will transmit only when the following three condition: i) the channel is sense idle. ii) $t<\Gamma$ (note that $\Gamma$ is in general different from the $\Gamma *$ that maximizes capacity), and iii) the SU queue length $\mathrm{M}$ is greater than the zero. The time threshold $\Gamma$ should be adjusted to satisfy the PU collision probability constraint. In Figure 14, examine the effect of busy distribution on $W$ and $\mathrm{p}_{\mathrm{c}}$. In which four different busy distributions with the same mean: exponential, uniform, Weibull, and the fixed busy time are considered respectively. Figure 14(a) shows that the busy distribution affects $W$ significantly. The exponential busy distribution and the fixed busy distribution induce the largest delay and the smallest delay, respectively. On the other hand, for the same $\Gamma$, Figure 14(b) shows that $p_{\mathrm{c}}$ changes only slightly with busy distributions. 


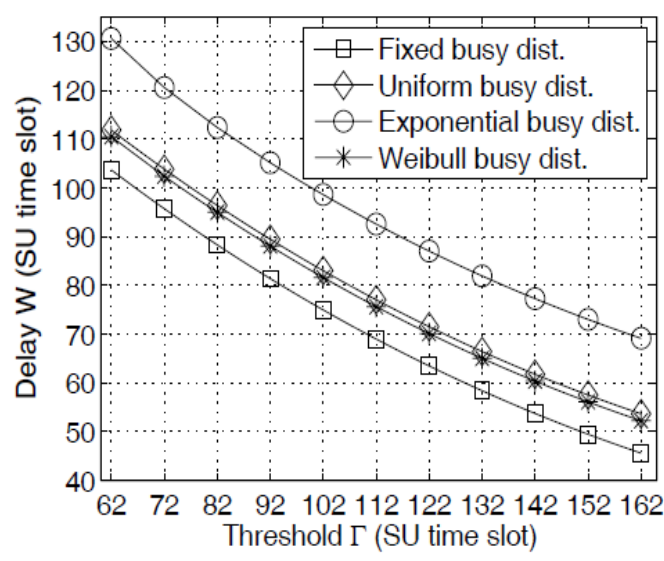

(a) Delay

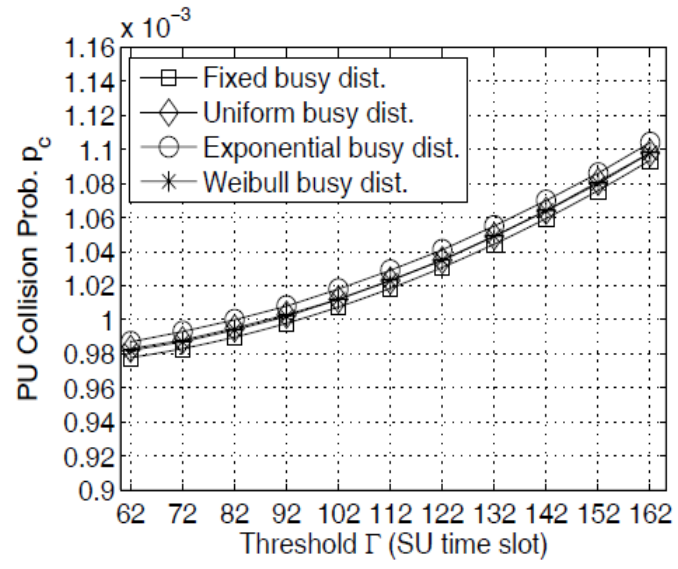

(b) Collision probability

Figure 14 Performance of threshold policies as a function of $\Gamma$. Delay and collision probability Comparison of various busy distributions. Assume a uniform idle distribution and $\mathrm{p}=0.11$.

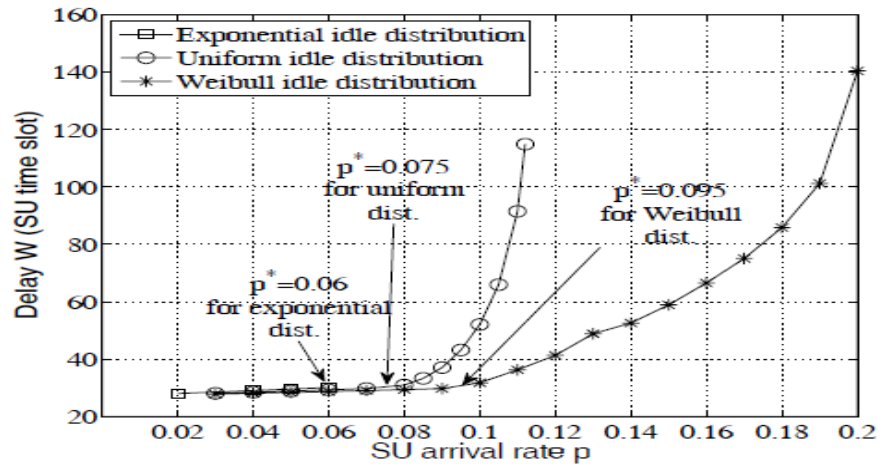

Figure 15: Delay performance of threshold policies as a function of $p$.

Figure 15 shows the delay of the threshold policy as a function p. For each $p$, choose $\Gamma$ of the threshold policy such that $\eta=0.001$ a uniform busy distribution and various idle distributions. For each idle distribution, there exists some $p *$ such that when $p<p *$, the threshold policy becomes the greedy policy. Find that $p *=0.06,0.075,0.095$ for the exponential, uniform, and Weibull distribution, respectively. Figure 15 shows that for these idle distributions, the delay of the threshold policy is similar in the region $p<p *$ where the greedy policy is optimal. For the exponential distribution, $p *=0.06$ is the highest arrival rate such that $p c$ does not exceed $\eta$. In comparison the capacity of the uniform distribution is higher. Therefore, when $p \in[0.075$, 0.114], a threshold policy can be found to ensure that $p_{c}=\eta$ at the rate of increased delay. Thus that the delay of the threshold policy increases rapidly as $p$ approaches $C=0.114$. The Weibull distribution has the highest capacity. When $p \in[0.095,0.2]$, the delay of the threshold policy increases with $p$, but at a slower rate than that of the uniform distribution. The accuracy of the above graph has been confirmed for several commonly used busy and idle distributions. This method is shown that the busy time distribution significant impact of delay of the SU, while the idle distribution largely determines the transmission threshold and collision probability. 


\section{vi) Fuzzy logic Based System}

Fuzzy logic based spectrum access method is proposed in [23]. Fuzzy logic is a multi-valued logic. It uses many input parameters to take the decision. Here distance, signal strength, velocity and spectrum efficiency are considered as input parameters. If the signal strength of the intended channel is high ratio will decide to change of the channel. When the node velocity is more, more will be the chance for a mobile node to change position. The ratio of the required spectrum by the secondary user to the total available spectrum (spectrum efficiency) is determining parameter in this parameter dynamic spectrum access policy radio will use unused vacant spectrum. The distance between the primary licensed and secondary unlicensed user has been one of the determining parameters in which the secondary user at a closer distance to the licensed primary user, give priority to access spectrum. Simulation results show that [23], the chance of taking decision increases if the signal strength of the channel offered by primary user is high and distance between primary and secondary users is low as shown in Figure 16. As the velocity increases the chance of the spectrum accessing is more if the distance is small as shown in Figure 17.

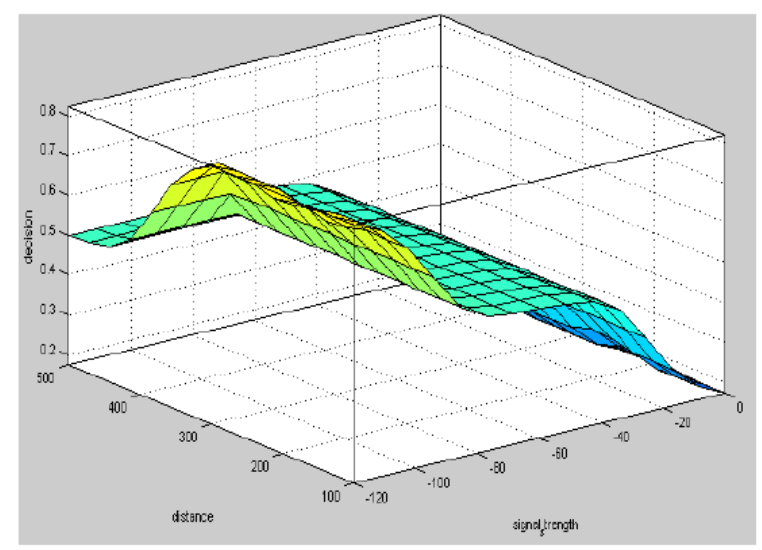

Figure 16 Opportunistically spectrum access decision possibility (velocity $=50 \mathrm{Km} / \mathrm{hr}$ and ratio of the required spectrum to available spectrum $=0.5$ )

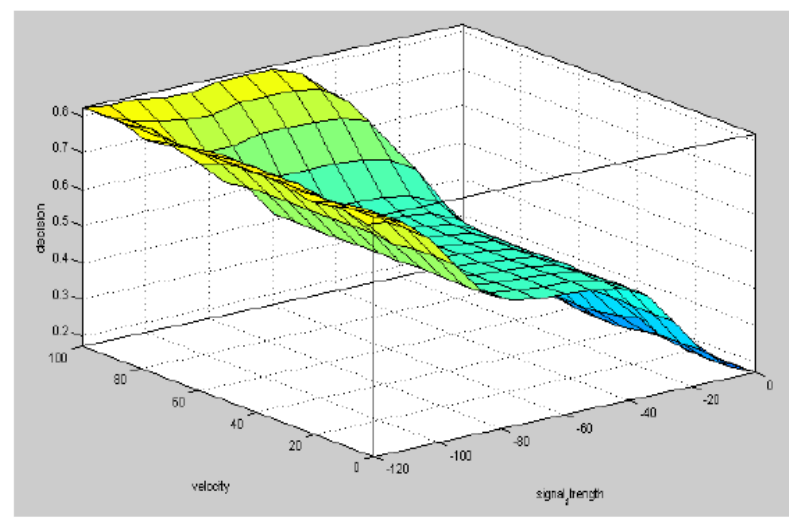

Figure 17 Opportunistically spectrum access decision possibility (Distance between the primary and secondary user $=50$ meters and user velocity $=50 \mathrm{Km} / \mathrm{hr}$ ) 


\section{vii) Spatio-Temporal Spectrum Management Model}

Spatio-Temporal Spectrum Management Model is used for coordinating DSA networks [24]. This Model is reducing complexity of the problem into temporal dynamic spectrum allocation problem and spatial dynamic spectrum allocation problem. In this model regional spectrum broker (RSB) coordinated the temporal dynamic spectrum allocation for a given region within the assumed spatial distribution of the spectrum demand is homogeneous. Spectrum Broker Coordinator (SBC) is a centralized entity, which stores the spectrum demands of the regions, and spectrum management at the border of the regions is realized based on this information. Architecture of the model is shown in Figure 18.

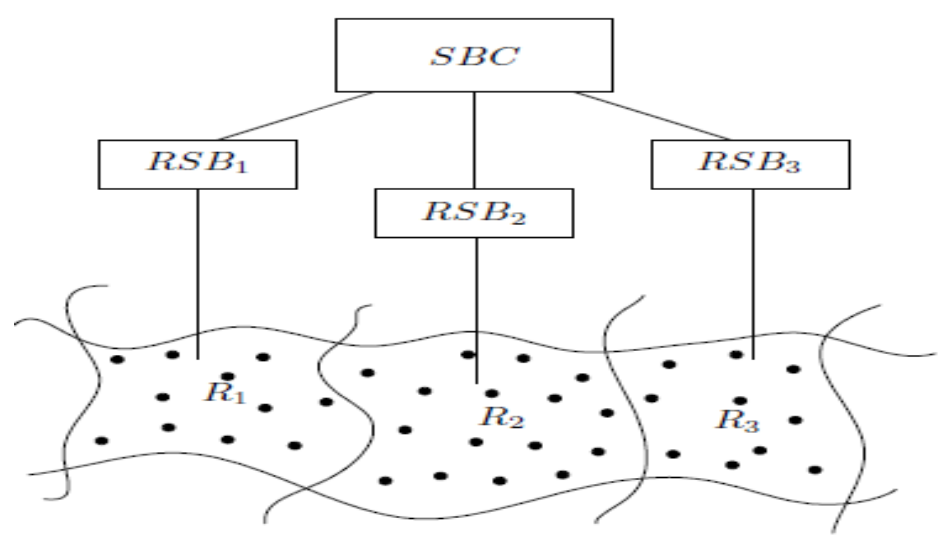

Figure 18 Spatio-Temporal DSA architecture

In TDSA method the service providers of the region send their demands for spectrum to RSB. The RSB allocates continuous spectrum blocks to the requesters separated by guard bands. The size of the blocks may vary in time. Moreover demanding another spectrum block, service providers are returning spectrum blocks that they do not need. The requests are batch-processed at given time-intervals.

The Spectrum Dynamic Spectrum Allocation (SDSA) handles spectrum demands arising at the same time in different regions. The main objective of the SDSA is attuning the different demands within different regions on the way, where the least interference arises in the overlapping regions. In order to realize this, the RSB needs to have information about the actual spectrum allocation of the neighboring regions. To collect this information, a time snapshot of the spectrum usages inside a region is sent by the RSBs to the Spectrum Broker Coordinator (SBC) Based on this information, the RSBs can manage the problem of interference in the overlapping regions accordingly. The hierarchy of the RSBs and SBC is shown in Figure 18. Simulation results are shown in Figure 19 and Figure 20. In region 1 the demand of $\mathrm{NSP}_{1}$ is much larger than that of the region $\mathrm{NSP}_{2}$, in region 2 , it was just opposite way. The numbers of carriers used by the providers in the both regions as a function of time .The excess spectrum required to fulfill the demands in the case of overhearing is denoted by a darker tone shown in Figure. 


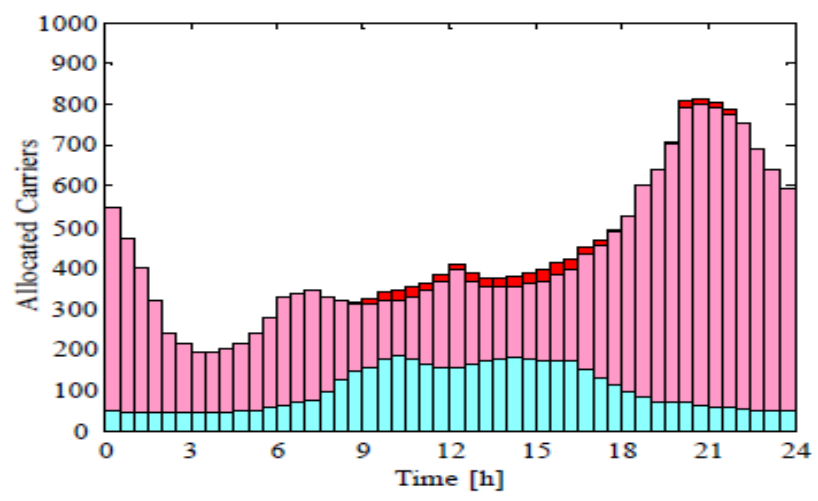

Figure 19 Allocated spectrum sizes for both providers in region 1.

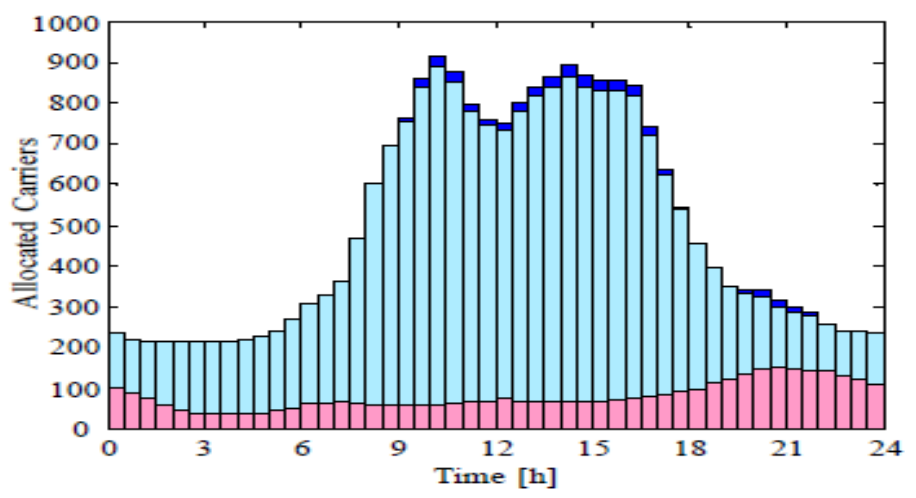

Figure 20 Allocated spectrum sizes for both providers in region 2

\section{CONCLUSIONS}

Cognitive radio is an emerging technology, which can provide faster and more reliable wireless service by utilizing the existing spectrum band more efficient. Here discussed different dynamic spectrum access models. Game theory is an important tool in studying, modeling and analyzing the Cognitive interaction process. A Measurement based model in which the continuous time semi Markov model is used. Continuous-time semi Markov model captures the idle periods remaining between the bursty transmissions of a wireless LAN. This model strikes a good compromise between accuracy and computation complexity. DSA using a NC4 method opportunistically and efficiently accesses the available channel for communication. In the Markovian queuing model a centralized architecture is proposed for allocation of bandwidth and to find blocking probability. The delay performance of threshold policies is to minimize the delay of SU subject to a PU collision probability constraint. A fuzzy logic based spectrum management technique help to take wise decision with respect to spectrum sharing in cognitive radio networks. Spatio-Temporal spectrum management model simplify the spectrum allocation problem and described architecture that splits the complex problem into temporal and spatial parts. 


\section{References:}

[1] http://searchnetworking.techtarget.com/resource definition from WhatIs_com.htm, "What is cognitive radio (CR)?"

[2] www.wikipedia.org.

[3] K. -C. Chen, Y. -J. Peng, N. Prasad and Y. -C. Liang, S. Sun, " Cognitive Radio Network Architecture: Part I - General Structure", Proceeding of the ACM International Conference on Ubiquitous Information Management and Communication, Seoul, 2008

[4] Przemyslaw Pawelczak "Cognitive Radio: From Utopia to Reality" Freeband Ambient Communication Event, Enscheda, 4 July 2006

[5] Anita Garhwal1 and Partha Pratim Bhattacharya2 "A Survey on Dynamic spectrum Access techniques for cognitive radio" International Journal of Next-Generation Networks (IJNGN) Vol.3, No.4, December 2011 pp.15-32.

[6] Rahul Tandra, Shridhar Mubaraq Mishra and Anant Sahai "What is a spectrum hole and what does it take to recognize one?” IEEE Journal and Magazine, Vol.97, issue 5, May 2009, pp 1-22.

[7] Anita Garhwal, Partha Pratim Bhattacharya, "Dynamic Spectrum Access in Cognitive Radio: a brief review", International Journal of Computer Application in Engineering Sciences, Special Issue on Computer Networks \& Security, Vol.1 December 2011, pp. 149-153.

[8] J. Mitola III and G. Maquire (1999), "Cognitive Radio Making Software Radios More Personal”,IEEE Pres. Comm.,6(4), 1999, pp 13-18.

[9] Mansi Subhedar1 and Gajanan Birajdar2 "Spectrum Sensing Technique in Cognitive Radio Network Spectrum Access Networks: A Survey” International Journal of Next-Generation Networks (IJNGN) Vol.3, No.2, June 2011 pp. 37-51.

[10] Alice Crohas "Practical Implementation of a Cognitive Radio System for Dynamic Spectrum Access" July2008pp 1-46.

[11] Przemyslaw s Pawelczak, Gerard J.M Janssen and R.Venkatesha Prasad "Performance Measures of Dynamic Spectrum Access Networks”IEEE Globecom, 28 November 2006 pp.1-22.

[12] http://www.mwrf.com/

[13] Brief note "Dynamic spectrum access" commission for communication regulation document no.7/22, 13 April 2007 pp.1-20.

[14] Qings Zhao and Brian M. Sadler “A Survey of Dynamic Spectrum Access” IEEE Signal Processing Magazine, May 2007 pp.79-89.

[15] Xavier Gelabert "Cognitive Radio Enabling Opportunistic Spectrum Access (OSA): Challenges and Modeling Approaches" UPF-DTIC Weekly Research seminar 28 October 2010.

[16] Sudhir Srinivasa and Syed Ali Jafar "The Throughput Potential of Cognitive Radio: A Theoretical Perspective" IEEE Communications Magazine, May 2007 pp.73-79.

[17] Danda B. Rawat and Gongjun Yan "Spectrum Sensing Methods and Dynamic Spectrum Sharing in Cognitive Radio Networks: A Survey" International Journal of Research and Reviews in Wireless Sensor Networks Vol. 1, No. 1, March 2011.

[18] Beibei Wang, Yongle Wu, K.J. Ray Liu "Game theory for cognitive radio networks: An overview" Computer Networks 4, 13 April 2010 pp. 2537-2561.

[19] Stefan Geirhofer, Lang Tong and Brian M. sadler "Measurement-Based Model for Dynamic Spectrum Access in WLAN Channel" communication and network consortium 19 July 2011.

[20] Nicola Baldo, Alfred Asterjadhi and Michele Zorzi "Dynamic Spectrum Access Using a Network Coded Cognitive Control Channel” IEEE Transaction On Wireless Communication, Vol. 9, NO. 8, August 2010 pp. 2575 -2587. 
[21] Prabhjot Kaur, Arun Khosla and Moin Uddin "Markovian Queuing Model for Dynamic Spectrum Allocation in Centralized Architecture for Cognitive Radios" IACSIT International Journal of Engineering and Technology, Vol.3, No.1, February 2011 ISSN: 1793-8236.

[22] Rong-Rong Chen and Xin Liu "Delay Performance of Threshold Policies for Dynamic Spectrum Access" IEEE Transactions on Wireless Communication, VOL. 10, NO. 7, July 2011, pp.2283-2293.

[23] Partha Pratim Bhattacharya, Ronak Khandelwal, Rishita Gera, Anjali Agarwal, "Smart Radio Spectrum Management for Cognitive Radio", International Journal of Distributed and Parallel Systems, Vol. 2, no. 4, July 2011

[24] L'aszl 'o Kov'acs and Attila Vid'acs "SpatioTemporal Spectrum Management Model for Dynamic Spectrum Access Networks" Published by ACM August 2006, Proceedings of the first international workshop on Technology and policy for accessing spectrum (TAPAS).

\section{Authors}

Pinki Yadav was born in India on February 2, 1987. She received her B.E. Degree in Electronics and Communication Engineering from Mandsaur Institute of Technology, Mandsaur (University RGPV Bhopal), M.P., India in 2010 and currently is an M. Tech (Signal Processing) student in Mody Institute of Technology and Science (Deemed University), Rajasthan, India.

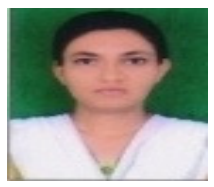

Subhajit Chatterjee was born in India. He has more than 12 years experience in teaching starting and 3 years in industry. He has served some reputed educational institutes in different positions from Lecturer to Teacher In Charge of department. Presently he is working as Assistant Professor, Department of Electronics \& Communication Engineering, Regent Education and Research Foundation, Barackpore. $\mathrm{He}$ has number of publications and his area of interests are Analog \& Digital

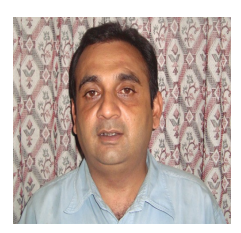
Communications, Solid State Devices, Telecommunication, Microprocessors \& Microcontrollers. He has co authored two books on Microprocessors \& Microcontrollers and Telecommunication \& Switching respectively. He has reviewed chapters of books published by reputed publishers.Presently he is doing his research on Spectrum Access in Cognitive Radio.

Dr. Partha Pratim Bhattacharya was born in India on January 3, 1971. He has 15 years of experience in teaching and research. He served many reputed educational Institutes In India in various positions starting from Lecturer to Professor and Principal. At present he is working as Professor in Department of Electronics and Communication Engineering in the Faculty of Engineering and Technology, Mody Institute of Technology and Science (Deemed University), Rajasthan, India. He worked on Microwave devices and systems and mobile cellular communication systems. He has published a good number of papers in

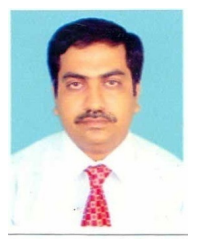
refereed journals and conferences. His present research interest includes mobile communication and cognitive radio. Dr. Bhattacharya is a member of The Institution of Electronics and Telecommunication Engineers, India and The Institution of Engineers, India. He is the recipient of Young Scientist Award from International Union of Radio Science in 2005. He is working as the chief editor, editorial board member and reviewer in many reputed journals. 